\title{
Influence of Primary Care on Breast Cancer Outcomes Among Medicare Beneficiaries
}

\author{
Richard G. Roetzheim, MD, MSPH', \\ Jeanne M. Ferrante, MD, MPH ${ }^{3,4}$ \\ Ji-Hyun Lee, DrPH ${ }^{2}$ \\ Ren Chen, MD, MPH \\ Kymia M. Love-Jackson, MBA ${ }^{1}$ \\ Eduardo C. Gonzalez, $M D^{1}$ \\ Kate J. Fisher, $M A^{2}$ \\ Ellen P. McCartby, PbD, MPH
}

'Department of Family Medicine, Morsani College of Medicine, University of South Florida, Tampa, Florida

${ }^{2} \mathrm{H}$. Lee Moffitt Cancer Center and Research Institute, Tampa, Florida

${ }^{3}$ Department of Family Medicine and Community Health, University of Medicine and Dentistry of New Jersey-Robert Wood Johnson Medical School, Newark, New Jersey

${ }^{4}$ Cancer Institute of New Jersey, New Brunswick, New Jersey

${ }^{5}$ Division of General Medicine and Primary Care, Department of Medicine, Harvard Medical School, Beth Israel Deaconess Medical Center, Boston, Massachusetts

Conflicts of interest: authors report none.

\section{CORRESPONDING AUTHOR}

Richard Roetzheim, MD, MSPH Department of Family Medicine Morsani College of Medicine University of South Florida 12901 Bruce B. Downs Blvd, MDC 13

Tampa, FL 33612

rroetzhe@health.usf.edu

\begin{abstract}
PURPOSE We used the Surveillance Epidemiology and End Results (SEER)-Medicare database to explore the association between primary care and breast cancer outcomes.
\end{abstract}

METHODS Using a retrospective cohort study of 105,105 female Medicare beneficiaries with a diagnosis of breast cancer in SEER registries during the years 1994-2005, we examined the total number of office visits to primary care physicians and non-primary care physicians in a 24-month period before cancer diagnosis. For women with invasive cancers, we examined the odds of diagnosis of late-stage disease, according to the American Joint Commission on Cancer (AJCC) (stages III and IV vs stages I and II), and survival (breast cancer specific and all cause) using logistic regression and proportional hazards models, respectively. We also explored whether including noninvasive cancers, such as ductal carcinoma in situ (DCIS), would alter results and whether prior mammography was a potential mediator of associations.

RESULTS Primary care physician visits were associated with improved breast cancer outcomes, including greater use of mammography, reduced odds of latestage diagnosis, and lower breast cancer and overall mortality. Prior mammography (and resultant earlier stage diagnosis) mediated these associations in part, but not completely. Similar results were seen for non-primary care physician visits. Results were similar when women with DCIS were included in the analysis.

CONCLUSIONS Medicare beneficiaries with breast cancer had better outcomes if they made greater use of a primary care physician's ambulatory services. These findings suggest adequate primary medical care may be an important factor in achieving optimal breast cancer outcomes.

Ann Fam Med 2012;10:401-411. doi:10.1370/afm.1398

\section{INTRODUCTION}

$\mathrm{P}$ rimary care physicians play an important role in early cancer detection. A recommendation from a primary care physician, for example, is consistently one of the strongest predictors of cancer screening. ${ }^{1-10}$ Patients who are referred to or are assigned a primary care physician are more likely to undergo most forms of cancer screening, including mammograms ${ }^{11-13}$ and clinical breast examinations. ${ }^{11}$ Primary care physicians may also ensure more timely diagnosis after screening abnormalities and prevent diagnostic delay. ${ }^{14}$

Understanding the effects of primary medical care is important because a fundamental shift is occurring in our nation's health care system. Since 1970 there has been marked growth in the number of specialist physicians, with primary care making up a progressively lower proportion of the overall physician workforce..$^{15,16}$ In most nations, primary care physicians account for $50 \%$ of the physician workforce..$^{17}$ In the United States, however, they now make up less than one-third of all physicians, and their proportion is expected to decline to about $25 \%$ in the next 10 years. ${ }^{18}$ The number of medical students interested in primary care careers has also 
progressively declined, ${ }^{15,19-23}$ and only $7 \%$ of graduating medical students plan careers in adult primary care. ${ }^{24}$ If these trends continue, the decline in our nation's primary health care system may have important implications for the goal of improving cancer-related outcomes.

Ecologic studies have found that a higher primary care physician supply is associated with improved health outcomes, such as overall mortality; cardiovascular, infant, and cancer mortality; and earlier cancer stage at diagnosis. ${ }^{25-38}$ All these studies are subject to the ecological fallacy, however; it is not possible to determine whether individuals with better outcomes in these studies are the same individuals who received care from primary care physicians.

Despite the important role played by primary care physicians in preventive care, the actual effect of primary medical care on cancer outcomes has not been well studied. The degree to which primary medical care affects outcomes, such as stage at diagnosis and cancer mortality, is uncertain. Understanding the relationship between primary medical care and cancer outcomes is important in preparing for the major changes that are occurring to our nation's health care system.

\section{METHODS}

This study utilized a retrospective cohort design to assess primary care utilization and cancer outcomes. The 282,869 women with a diagnosis of breast cancer within the Surveillance Epidemiology and End Results (SEER)-Medicare linked data set during the years 1994 to 2005 represented out cohort of interest. This sample does not include women with premalignant lesions, such as lobular carcinoma in situ. We placed exclusions on the analytic sample to ensure that relevant clinical information was available and to help control for selection bias and confounding by unmeasured factors. Because of clinical complexity, we excluded women who were eligible for Medicare because of end-stage renal disease $(n=897)$ and women whose first SEER-registered cancer was not actually their first cancer diagnosis $(n=5,945)$. We then excluded women whose first diagnosis was not breast cancer and women who developed a type of cancer other than breast within 1 year of their first diagnosis $(n=11,948)$. A 24-month period was required to provide a stable estimate of physician visits. To ensure that patients had at least 24 months of Medicare claims before their cancer diagnosis, we excluded women with breast cancer diagnosed before the age of 67 years $(n=102,582)$. We also excluded breast cancer cases in which the diagnosis was based solely on death certificates or at autopsy $(n=1,936)$. Because patients enrolled in a Medicare health maintenance organization
(HMO) have no claims history, we excluded all women enrolled in a Medicare HMO during the year of cancer diagnosis and the year before diagnosis $(n=42,429)$. For similar reasons, we excluded women that did not have continuous Part A and Part B Medicare coverage during this 2 -year period $(n=12,027)$. The final analytic sample included 105,105 women.

There is considerable empiric evidence that preventive care in general and cancer screening in particular are health services that are overwhelmingly delivered in the ambulatory setting. ${ }^{39-48}$ We therefore examined Medicare claims (National Claims History, NCH) for the following ambulatory-based evaluation and management services representing routine office visits: 99201-99205, 99211-99215.

Similar to earlier research, we identified the physician specialty associated with each claim using the unique physician identification number and the Medicare provider specialty field found in NCH claims. ${ }^{49,50}$ We defined primary care physicians as having the following specialties: general practice, family medicine, primary care internal medicine, geriatric medicine, obstetrics-gynecology, and physicians practicing in multispecialty group practices without a defined specialty. The distribution of physician visits by specialty was as follows: general practice $9.5 \%$, family medicine $26.8 \%$, primary care internal medicine $50.0 \%$, geriatric medicine $0.5 \%$, obstetrics-gynecology $6.0 \%$, and multispecialty group practice $7.1 \%$. For each woman, we assessed primary care and non-primary care physician office visits during a 24 -month period before the cancer diagnosis.

Because physician visit patterns are likely to change during the time that a potential cancer is being diagnosed, we excluded the 3-month period immediately before diagnosis and assessed physician claims during the 24 months before this period (ie, the 3 to 27 months before diagnosis). ${ }^{51}$ Previous studies suggest that the overwhelming majority of patients complete diagnostic evaluations within this time. ${ }^{14,52}$

Stage at diagnosis was classified using the American Joint Commission on Cancer (AJCC) staging system $(0$, I, II, III, and IV). The SEER database provided data on vital status and underlying cause of death for all patients through 2005. The following variables were used as potential confounders in multivariable models; age at diagnosis, race-ethnicity, marital status at diagnosis, census-derived measures of median household income (categorized by quintiles within each registry), education attainment (percentage of persons with less than a high school education, categorized by quintiles within each registry), metropolitan statistical area, influenza vaccination (as a marker of preventive behaviors), year of diagnosis, SEER geographic registry, 
Charlson comorbidity index ${ }^{53}$ (determined from both inpatient and outpatient claims), and histologic cancer type categorized as ductal, lobular, combined ductal/ lobular, favorable (medullary, tubular, papillary, mucinous), or unfavorable (inflammatory, Paget's).

We examined the relationship between primary care and non-primary care physician ambulatory claims and likelihood of late-stage diagnosis (stage III, IV vs stage I, II) using multivariable logistic regression. Because the malignant potential of ductal carcinoma in situ (DCIS) is uncertain, we first examined only women having invasive breast cancer (in situ cases excluded), and then repeated our analysis including women with DCIS. We excluded women whose cancer stage was unknown at diagnosis from these analyses $(n=10,694)$. We also examined whether an unknown stage was related to measures of primary care physician visits. We estimated odds ratios of late-stage diagnosis and its $95 \%$ confidence intervals, comparing each category of physician utilization to its referent group.

As one probable mechanism by which primary care would lead to earlier diagnosis, we also explored whether the primary care physician visits were associated with greater likelihood of receiving a mammogram in the 2-year period before diagnosis. To avoid capturing tests performed at the time of breast cancer diagnosis, we assessed whether women had any bilateral mammogram in the 2-year period from 3 months to 27 months before diagnosis. ${ }^{54}$ We did not try to differentiate screening and diagnostic mammograms because of the inherent difficulty in distinguishing this information from claims data. ${ }^{55,56}$ To determine whether physician visits were associated with late-stage diagnosis above and beyond receipt of a mammogram, we reran logistic models including a variable for a prior mammogram.

We examined mortality (all-cause and breast cancer mortality) among women having invasive breast cancers (in situ cancers were excluded, but cancers missing a diagnostic stage were included). The SEER-Medicare database provided vital status through December 31,2007 , and information on specific cause of death through December 31, 2005. All-cause mortality was therefore assessed from date of diagnosis to date of death or last known follow-up (December 31, 2007). Mortality from breast cancer was assessed from date of diagnosis through December 31, 2005. Among the 90,537 women having invasive breast cancer (including those with an unknown diagnostic stage), there were 35,496 deaths overall and 8,898 deaths due to breast cancer during the follow-up period. In all-cause mortality analyses, women who were alive at the end of

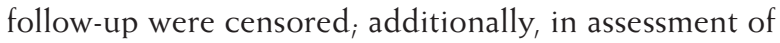
breast cancer mortality, those who died of causes other than breast cancer were also censored.
We also analyzed mortality using Cox proportional regression modeling, adjusting for potential confounding factors described above with further adjustment for tumor characteristics that may affect mortality (tumor grade, estrogen/progesterone receptor status, tumor size as a continuous variable). To determine whether associations between primary care physician visits and lower breast cancer mortality were primarily the result of earlier stage at diagnosis, hazard models were first performed excluding stage at diagnosis and tumor size and then repeated with stage (including an indicator variable for a missing stage) and tumor size.

\section{RESULTS}

Table 1 describes the final analytic sample. The mean age of the sample was 76.5 years (median 76 years, range 67 to 107 years, SD, 6.6 years). Most breast cancers were either ductal or lobular, and most women $(80.9 \%)$ had early-stage (AJCC 0, I, II) disease. Women had on average 14 physician office visits (both primary care and non-primary care) in the 24-month prediagnostic period ( $\mathrm{SD}, 13$, median 11 visits). Primary care and non-primary care physician visits tended to be correlated (Spearman correlation coefficient $0.15, P<.001$ ).

There were $57,769(55 \%)$ women who had a mammogram in the 3 - to 27 -month period before a cancer diagnosis $(83.7 \%$ of mammograms were ordered by a primary care physician). Table 2 describes predictors of mammography in the 3-to 27 -month period before a cancer diagnosis for women having invasive breast cancers (stage I to IV, or unknown staging). The odds of a prior mammogram increased with an increasing number of primary care physician visits. Women who had more than 10 visits had 4.0 times greater odds of prior mammography compared with women having 0 or 1 encounter. The odds of prior mammography also increased with an increasing number of non-primary care physician visits in a similar fashion, with women who had more than 10 visits having 3.3 times greater odds of prior mammography compared with women having 0 or 1 encounter.

Table 3 shows the relationship between ambulatory visits and the odds of late AJCC stage disease (III, IV) at diagnosis. The odds of late-stage disease decreased with increasing number of ambulatory primary care and non-primary care physician visits. Women having 11 or more primary care visits had $50 \%$ lower odds of late-stage disease compared with women having 0 or 1 primary care visit.

Prior mammography was associated with reduced odds of late-stage disease (adjusted odds ratio 0.36; $95 \% \mathrm{CI}, 0.34-0.38, P<.001)$. The association between primary care physician visits and late-stage disease was also attenuated when a variable for prior mam- 
mogram was included in the logistic models (adjusted odds ratios [AORs] for primary care physician visits: 0 to 1 visit, $\mathrm{AOR}=1.0$ [referent]; 2 to 4 visits, $A O R=0.68[95 \%$ CI, 0.64-0.73]; 5 to 10 visits, $\mathrm{AOR}=0.62[95 \% \mathrm{CI}, 0.59$ $0.66]_{i} \geq 11$ visits, $\mathrm{AOR}=0.65$ [95\% CI, 0.61-0.69]).

Table 4 presents the relationship between ambulatory visits and breast cancer mortality. In unadjusted analysis and analysis adjusting for confounders other than stage and tumor size, an increase in both primary care and non-primary care physician visits was associated with lower breast cancer mortality. Women having more than 10 primary care physician visits had $41 \%$ lower breast cancer mortality compared with women having 0 to 1 visit when adjusting for confounders other than stage and size. Results were attenuated when stage at diagnosis and tumor size were included in logistic models, although these relationships persisted. All-cause mortality also diminished with increasing numbers of primary care physician visits (adjusted hazard ratios [AHRs]: 0 to 1 visit, $\mathrm{AHR}=1.0$ [referent group]; 2 to 4 visits, $\mathrm{AHR}=0.76[95 \%$ $\mathrm{CI}, 0.73-0.78] ; 5$ to 10 visits, $\mathrm{AHR}=0.70[95 \% \mathrm{CI}, 0.68-$ $0.73]_{i} \geq 11$ visits, $\mathrm{AHR}=0.73$ [95\% CI, 0.70-0.75]).

Results were similar when analyses were repeated with DCIS cases included. Associations between primary care physician visits and cancer outcomes were also similar when analyzed separately within categories of non-primary care physician utilization ( 0 to 1,2 to 4,5 to $10, \geq 11$ visits).

\section{DISCUSSION}

We found that an increasing number of primary care physician office visits were associated with improved $(\mathrm{N}=105,105)$
Table 1. Characteristics of Women With a Diagnosis of Breast Cancer

\begin{tabular}{|c|c|c|c|}
\hline Characteristic & Value & Characteristic & Value \\
\hline \multirow{2}{*}{$\begin{array}{l}\text { Total ambulatory primary care } \\
\text { physician visits, } \mathrm{n}(\%)\end{array}$} & & \multicolumn{2}{|l|}{ Histologic type, n (\%) } \\
\hline & & Ductal & $74,760(71.1)$ \\
\hline 0-1 & $21,469(20.4)$ & Lobular & $17,176(16.3)$ \\
\hline $2-4$ & $19,355(18.4)$ & Ductal/lobular & $347(0.3)$ \\
\hline $5-10$ & $33,364(31.7)$ & Favorable & $5,961(5.7)$ \\
\hline$\geq 11$ & $30,917(29.4)$ & Unfavorable & $1,315(1.3)$ \\
\hline \multicolumn{2}{|l|}{$\begin{array}{l}\text { Total ambulatory non-primary } \\
\text { care physician visits, } \mathrm{n}(\%)\end{array}$} & Undefined & $5,546(5.3)$ \\
\hline $0-1$ & $39,031(37.1)$ & \multicolumn{2}{|l|}{$\begin{array}{l}\text { Estrogen receptors, } \\
n(\%)\end{array}$} \\
\hline $2-4$ & $23,308(22.2)$ & Positive/borderline & $49,010(46.6)$ \\
\hline $5-10$ & $23,230(22.1)$ & Negative & $9,147(8.7)$ \\
\hline$\geq 11$ & $19,536(18.6)$ & Missing & $46,948(44.7)$ \\
\hline \multicolumn{2}{|l|}{ Age at diagnosis, y (\%) } & \multicolumn{2}{|l|}{$\begin{array}{l}\text { Progesterone receptor, } \\
\mathrm{n}(\%)\end{array}$} \\
\hline $76-85$ & $42,913(40.83)$ & Positive/borderline & $40,265(38.3)$ \\
\hline$\geq 86$ & 10,902 (10.37) & Negative & $17,027(16.2)$ \\
\hline \multicolumn{2}{|l|}{ Race/ethnicity, n (\%) } & Missing & $47,813(45.5$ \\
\hline White, non-Hispanic & $90,372(86.0)$ & \multicolumn{2}{|l|}{ Tumor size, mm } \\
\hline Black, non-Hispanic & $6,694(6.4)$ & Mean (SD) & $20.2(20.6)$ \\
\hline Hispanic & $3,931(3.7)$ & \multirow{3}{*}{\multicolumn{2}{|c|}{$\begin{array}{l}\text { Median (minimum- } \\
\text { maximum) } \\
\text { AJCC stage at diagno- } \\
\text { sis, } n(\%)\end{array}$}} \\
\hline $\begin{array}{l}\text { Asian/American Indian/ } \\
\text { Pacific Islander }\end{array}$ & $3,425(3.3)$ & & \\
\hline Other & $683(0.7)$ & & \\
\hline \multicolumn{2}{|l|}{ Marital status, n (\%) } & 0 (in situ) & $14,568(13.9)$ \\
\hline Single (never married) & $7,564(7.2)$ & I & $42,830(40.8)$ \\
\hline Married & $43,030(40.9)$ & II & $27,602(26.3)$ \\
\hline Separated/divorced & $6,913(6.6)$ & III & $6,012(5.7)$ \\
\hline Widowed & $43,280(41.2)$ & IV & $3,399(3.2)$ \\
\hline Unknown & $4,318(4.1)$ & Unknown & $10,694(10.2)$ \\
\hline MSA of residence, $\mathrm{n}(\%)$ & & \multicolumn{2}{|l|}{$\begin{array}{l}\text { Charlson comorbidity } \\
\text { index, } \mathrm{n}(\%)^{\mathrm{a}}\end{array}$} \\
\hline Large metropolitan & $60,122(57.2)$ & 0 & $61,466(58.5)$ \\
\hline Metropolitan & $29,272(27.9)$ & 1 & $24,979(23.8)$ \\
\hline Urban & $6,360(6.1)$ & $\geq 2$ & $18,660(17.8)$ \\
\hline Less urban & $7,632(7.3)$ & \multirow{2}{*}{\multicolumn{2}{|c|}{$\begin{array}{l}\text { Influenza vaccination, } \\
\text { n (\%) }\end{array}$}} \\
\hline Rural & $1,714(1.6)$ & & \\
\hline \multicolumn{2}{|l|}{ Year of diagnosis, $n(\%)$} & No & $46,673(44.4)$ \\
\hline 1994-1997 & $24,139(23.0)$ & \multirow[t]{3}{*}{ Yes } & \multirow[t]{3}{*}{$58,432(55.6)$} \\
\hline $1998-2000$ & $23,597(22.5)$ & & \\
\hline 2001-2005 & $57,369(54.6)$ & & \\
\hline
\end{tabular}

breast cancer outcomes. Women having 10 or more office visits were $50 \%$ less likely to have late-stage cancer diagnosed and had $41 \%$ lower breast cancer mortality, as well as $27 \%$ lower overall mortality, compared with women having 0 to 1 visit. Improved breast cancer outcomes among women with greater numbers of primary care physician office visits were in part, but not completely, explained by greater use of mammography and resultant earlier stage diagnosis.

A number of studies have linked the supply of primary care physicians to earlier stage of breast cancer 
at diagnosis, lower breast cancer mortality, and lower overall mortality. ${ }^{57-61}$ Research has also suggested a strong relationship between the receipt of primary medical care and recommendations for breast cancer screening. ${ }^{1,62,63}$ Our study confirms the findings of others that Medicare beneficiaries having greater numbers of primary care physician visits were more likely to receive mammography before diagnosis and were more likely to have early-stage breast cancer. ${ }^{61}$ Factors other than mammography might also mediate the association between primary care physician encounters and breast cancer stage. For example, having a primary care physician may lead to earlier diagnosis after the onset of breast cancer symptoms or an abnormal mammogram, ${ }^{14}$ or it may encourage healthier behaviors that affect breast cancer stage. ${ }^{64-66}$
The association between breast cancer mortality and visits to a primary care physician appeared to be mediated in large part by earlier stage at diagnosis. This association remained, however, even after controlling for stage, suggesting the possibility of additional factors associated with primary care. Potential roles of primary care after cancer diagnosis include assisting with treatment decisions, facilitating access to treatments, ensuring compliance with treatments and surveillance, and facilitating psychosocial care. ${ }^{67-72}$ Although it is possible such actions could influence breast cancer mortality, ${ }^{49}$ it is not certain whether such care is typical in primary care encounters. ${ }^{73,74}$

Visits to primary care physicians were also associated with lower overall mortality for women with diagnosed breast cancer. Potential mechanisms by

\section{Table 2. Predictors of Mammography $(n=90,537)$}

\begin{tabular}{|c|c|c|c|c|c|c|c|}
\hline Characteristic & $\begin{array}{l}\text { Adjusted } \\
\text { OR }^{\mathrm{a}}\end{array}$ & $\begin{array}{c}95 \% \\
\text { Wald CL }\end{array}$ & $\begin{array}{c}P \\
\text { Value }\end{array}$ & Characteristic & $\begin{array}{l}\text { Adjusted } \\
\text { OR }^{\mathrm{a}}\end{array}$ & $\begin{array}{c}95 \% \\
\text { Wald CL }\end{array}$ & $\begin{array}{c}P \\
\text { Value }\end{array}$ \\
\hline $\begin{array}{l}\text { Total ambulatory primary } \\
\text { care physician visits }\end{array}$ & & & & $\begin{array}{l}\text { Education level of } \\
\text { residence }\end{array}$ & & & \\
\hline 0-1 (referent) & 1.00 & - & - & Quintile 1 (lowest) & 1.00 & - & - \\
\hline $2-4$ & 2.78 & $2.65,2.92$ & $<.001$ & (referent) & & & \\
\hline $5-10$ & 3.67 & $3.51,3.83$ & $<.001$ & Quintile 2 & 1.04 & $0.99,1.09$ & 0.17 \\
\hline$\geq 11$ & 4.04 & $3.86,4.23$ & $<.001$ & Quintile 3 & 1.16 & $1.10,1.23$ & $<.001$ \\
\hline \multirow{3}{*}{$\begin{array}{l}\text { Total ambulatory non- } \\
\text { primary care physician } \\
\text { visits }\end{array}$} & & & & Quintile 4 & 1.23 & $1.16,1.30$ & $<.001$ \\
\hline & & & & Quintile 5 & 1.34 & $1.25,1.43$ & $<.001$ \\
\hline & & & & Income level of residence & & \multirow{3}{*}{-} & \multirow{3}{*}{-} \\
\hline 0-1 (referent) & 1.00 & - & - & \multirow{2}{*}{$\begin{array}{l}\text { Quintile } 1 \text { (lowest) } \\
\text { (referent) }\end{array}$} & \multirow[t]{2}{*}{1.00} & & \\
\hline $2-4$ & 1.72 & $1.65,1.78$ & $<.001$ & & & & \\
\hline $5-10$ & 2.15 & $2.06,2.23$ & $<.001$ & Quintile 2 & 0.99 & $0.94,1.04$ & .71 \\
\hline$\geq 11$ & 3.31 & $3.16,3.46$ & $<.001$ & Quintile 3 & 0.97 & $0.92,1.02$ & .26 \\
\hline \multicolumn{2}{|l|}{ Age at diagnosis } & & & Quintile 4 & 0.95 & $0.90,1.01$ & .13 \\
\hline $67-75$ y (referent) & 1.00 & & & Quintile 5 & 0.96 & $0.90,1.03$ & .28 \\
\hline $76-85$ y & 0.61 & $0.59,0.63$ & $<.001$ & MSA of residence & & & \\
\hline \multicolumn{2}{|l|}{ Race/ethnicity } & $0.24,0.27$ & $<.001$ & $\begin{array}{l}\text { Large metropolitan } \\
\text { (referent) }\end{array}$ & 1.00 & - & - \\
\hline \multirow{2}{*}{$\begin{array}{l}\text { White, non-Hispanic } \\
\text { (referent) }\end{array}$} & \multirow{2}{*}{1.00} & \multirow{2}{*}{ - } & \multirow{2}{*}{ - } & Metropolitan & 1.01 & $0.97,1.06$ & .55 \\
\hline & & & & Urban & 0.99 & $0.92,1.06$ & .73 \\
\hline Black, non-Hispanic & 1.01 & $0.95,1.08$ & .66 & Less urban & 0.93 & $0.86,1.00$ & .04 \\
\hline Hispanic & 0.80 & $0.73,0.86$ & $<.001$ & Rural & 1.05 & $0.92,1.19$ & .48 \\
\hline \multirow{2}{*}{$\begin{array}{l}\text { Asian/American } \\
\text { Indian/Pacific }\end{array}$} & 0.73 & $0.66,0.81$ & $<.001$ & \multicolumn{2}{|l|}{ Year of diagnosis } & & \\
\hline & & & & 1994-1997 (referent) & 1.00 & - & - \\
\hline Islander & & & & $1998-2000$ & 1.35 & $1.29,1.41$ & $<.001$ \\
\hline Other & 0.95 & $0.79,1.15$ & .62 & $2001-2005$ & 1.64 & $1.58,1.71$ & $<.001$ \\
\hline \multicolumn{4}{|l|}{ Marital status } & \multirow{2}{*}{\multicolumn{2}{|c|}{$\begin{array}{l}\text { Charlson comorbidity } \\
\text { index }\end{array}$}} & & \\
\hline Married (referent) & 1.00 & - & - & & & & \\
\hline Never married & 0.77 & $0.72,0.81$ & $<.001$ & 0 (referent) & 1.00 & - & - \\
\hline Separated/divorced & 0.71 & $0.67,0.75$ & $<.001$ & 1 & 0.74 & $0.72,0.77$ & $<.001$ \\
\hline Widowed & 0.72 & $0.69,0.74$ & $<.001$ & $\geq 2$ & 0.49 & $0.47,0.51$ & $<.001$ \\
\hline \multirow[t]{3}{*}{ Unknown } & 0.73 & $0.67,0.79$ & $<.001$ & \multicolumn{2}{|l|}{ Influenza vaccination } & & \\
\hline & & & & No (referent) & 1.00 & - & - \\
\hline & & & & Yes & 1.69 & $1.64,1.75$ & $<.001$ \\
\hline
\end{tabular}

$\mathrm{CL}=$ confidence limits; $\mathrm{MSA}=$ metropolitan statistical area.

Note: Patients with stage 0 carcinoma (in situ) were excluded.

a The multivariable logistic models also included indicator variables for specific cancer registry (data not presented). 
Table 3. Adjusted Associations of Late-Stage Diagnosis (AJCC III, IV) of Breast Cancer $(n=79,843$ )

\begin{tabular}{|c|c|c|c|c|c|c|c|}
\hline Characteristic & $\begin{array}{c}\text { Adjusted } \\
\text { OR }^{\mathrm{a}}\end{array}$ & $\begin{array}{c}95 \% \\
\text { Wald CL }\end{array}$ & $\begin{array}{c}P \\
\text { Value }\end{array}$ & Characteristic & $\begin{array}{l}\text { Adjusted } \\
\text { OR }^{a}\end{array}$ & $\begin{array}{c}95 \% \\
\text { Wald CL }\end{array}$ & $\begin{array}{c}P \\
\text { Value }\end{array}$ \\
\hline \multicolumn{4}{|l|}{$\begin{array}{l}\text { Total ambulatory primary } \\
\text { care physician visits }\end{array}$} & \multicolumn{4}{|l|}{$\begin{array}{l}\text { Education level of } \\
\text { residence }\end{array}$} \\
\hline 0-1 (referent) & 1.00 & - & - & Quintile 1 (lowest) & 1.00 & - & - \\
\hline $2-4$ & 0.57 & $0.53,0.61$ & $<.001$ & (referent) & & & \\
\hline $5-10$ & 0.49 & $0.46,0.52$ & $<.001$ & Quintile 2 & 0.98 & $0.91,1.05$ & .54 \\
\hline$\geq 11$ & 0.50 & $0.47,0.53$ & $<.001$ & Quintile 3 & 0.94 & $0.86,1.02$ & .12 \\
\hline \multirow{3}{*}{\multicolumn{4}{|c|}{$\begin{array}{l}\text { Total ambulatory non- } \\
\text { primary care physician } \\
\text { visits }\end{array}$}} & Quintile 4 & 0.87 & $0.79,0.95$ & .002 \\
\hline & & & & Quintile 5 & 0.81 & $0.73,0.90$ & $<.001$ \\
\hline & & & & \multicolumn{4}{|l|}{ Income level of residence } \\
\hline 0-1 (referent) & 1.00 & - & - & Quintile 1 (lowest) & 1.00 & - & - \\
\hline $2-4$ & 0.62 & $0.58,0.66$ & $<.001$ & (referent) & & & \\
\hline $5-10$ & 0.55 & $0.51,0.58$ & $<.001$ & Quintile 2 & 1.00 & $0.93,1.08$ & .90 \\
\hline$\geq 11$ & 0.46 & $0.42,0.49$ & $<.001$ & Quintile 3 & 1.05 & $0.97,1.14$ & .22 \\
\hline \multicolumn{4}{|l|}{ Age at diagnosis } & Quintile 4 & 1.08 & $0.99,1.19$ & .09 \\
\hline $67-75$ (referent) & 1.00 & - & - & Quintile 5 & 1.06 & $0.95,1.18$ & .28 \\
\hline $76-85$ & 1.21 & $1.15,1.27$ & $<.001$ & MSA of residence & & & \\
\hline$\geq 86$ & 1.67 & $1.55,1.79$ & $<.001$ & $\begin{array}{l}\text { Large metropolitan } \\
\text { (referent) }\end{array}$ & 1.00 & - & - \\
\hline \multicolumn{4}{|l|}{ Race/ethnicity } & Metropolitan & 0.97 & $0.91,1.04$ & 47 \\
\hline \multirow{2}{*}{$\begin{array}{l}\text { White, non-Hispanic } \\
\text { (referent) }\end{array}$} & \multirow[t]{2}{*}{1.00} & \multirow[t]{2}{*}{-} & \multirow[t]{2}{*}{-} & Urban & 0.96 & $0.86,1.08$ & $\begin{array}{r}.4 \angle \\
40\end{array}$ \\
\hline & & & & & & $0.00,1.00$ & .49 \\
\hline Black, non-Hispanic & 1.38 & $1.27,1.50$ & $<.001$ & Less urban & 0.84 & $0.75,0.95$ & .004 \\
\hline Hispanic & 1.32 & $1.19,1.48$ & $<.001$ & Rural & 0.83 & $0.68,1.00$ & .06 \\
\hline \multirow{2}{*}{$\begin{array}{l}\text { Asian/American } \\
\text { Indian/Pacific }\end{array}$} & 0.84 & $0.72,0.98$ & .03 & \multicolumn{4}{|l|}{ Year of diagnosis } \\
\hline & & & & 1994-1997 (referent) & 1.00 & - & - \\
\hline Islander & & & & $1998-2000$ & 0.90 & $0.84,0.97$ & .004 \\
\hline Other & 0.76 & $0.53,1.08$ & .13 & 2001-2005 & 1.09 & $1.02,1.16$ & .01 \\
\hline \multicolumn{4}{|l|}{ Marital status } & \multirow{2}{*}{\multicolumn{2}{|c|}{$\begin{array}{l}\text { Charlson comorbidity } \\
\text { index }\end{array}$}} & & \\
\hline Married (referent) & 1.00 & - & - & & & & \\
\hline Never married & 1.38 & $1.27,1.50$ & $<.001$ & 0 (referent) & 1.00 & - & - \\
\hline Separated/divorced & 1.25 & $1.14,1.37$ & $<.001$ & 1 & 1.02 & $0.96,1.08$ & .52 \\
\hline Widowed & 1.24 & $1.18,1.31$ & $<.001$ & $\geq 2$ & 1.21 & $1.13,1.29$ & $<.001$ \\
\hline \multirow[t]{3}{*}{ Unknown } & 1.23 & $1.08,1.39$ & .001 & \multicolumn{4}{|l|}{ Influenza vaccination } \\
\hline & & & & No (referent) & 1.00 & - & - \\
\hline & & & & Yes & 0.72 & $0.69,0.76$ & $<.001$ \\
\hline
\end{tabular}

$\mathrm{AJCC}=$ American Joint Commission on Cancer; $\mathrm{CL}=$ confidence limits; $\mathrm{MSA}=$ metropolitan statistical area; $\mathrm{PCP}=$ primary care physician.

Note: Patients with stage 0 carcinoma (in situ) or missing were excluded.

a Odds ratios indicate odds of late-stage (AJCC stages III, IV) diagnosis of breast cancer relative to early stage (AJCC stages I, II). Logistic models also include indicator variables for cancer registry (data not presented).

which primary care visits could affect overall mortality include promoting preventive services ${ }^{75,76}$ and managing comorbid illnesses, which occur in most cancer patients. ${ }^{77}$ Our study did not have sufficiently detailed data to assess these hypotheses, but our findings are consistent with a growing body of evidence linking primary care to improved cancer outcomes. ${ }^{28-31,34-36,38,61,78,79}$

Improved breast cancer outcomes were also seen for an increasing number of non-primary care physician visits. There are several possible explanations for the association between non-primary care physician visits and improved breast cancer outcomes. First, primary care and non-primary care physician visits tend to be correlated for such reasons as physician referrals and sharing common mediators of health care utilization (eg, health care needs of sicker patients). Although it is possible that some non-primary care physicians function in the role of primary care ${ }^{80}$ we found few mammograms ordered by non-primary care physicians, and we found equally strong associations in non-primary care specialties that traditionally have not functioned in that role (eg, ophthalmology, orthopedics, otolaryngology, data not shown). We also found that the associations for primary care physician visits were equally strong across all levels of utilization, suggesting that these non-primary care physician visits do not substitute for primary care encounters. Regardless of number of non-primary care visits, utilization of primary care physician visits confers independent 
Table 4. Adjusted Hazard Ratios for Breast Cancer Mortality $(n=90,537)$

\begin{tabular}{|c|c|c|c|}
\hline Characteristic & $\begin{array}{c}\text { Unadjusted } \\
\text { Hazard Ratio } \\
(95 \% \mathrm{Cl})\end{array}$ & $\begin{array}{l}\text { Adjusted Excluding } \\
\text { Stage and Tumor } \\
\text { Size }^{\mathrm{a}} \text { Hazard Ratio } \\
(95 \% \mathrm{Cl})\end{array}$ & $\begin{array}{c}\text { Adjusted Including } \\
\text { Stage and Tumor } \\
\text { Size }^{\text {a Hazard Ratio }} \\
(95 \% \mathrm{Cl})\end{array}$ \\
\hline \multicolumn{4}{|l|}{$\begin{array}{l}\text { Total ambulatory pri- } \\
\text { mary care physician } \\
\text { visits }\end{array}$} \\
\hline 0-1 (referent) & 1.00 & 1.00 & 1.00 \\
\hline $2-4$ & $0.55(0.52-0.59)$ & $0.68(0.64-0.73)$ & $0.89(0.82-0.95)$ \\
\hline $5-10$ & $0.50(0.47-0.53)$ & $0.61(0.58-0.65)$ & $0.83(0.77-0.89)$ \\
\hline$\geq 11$ & $0.50(0.47-0.53)$ & $0.59(0.55-0.63)$ & $0.80(0.74-0.86)$ \\
\hline \multicolumn{4}{|l|}{$\begin{array}{l}\text { Total ambulatory non- } \\
\text { primary care physi- } \\
\text { cian visits }\end{array}$} \\
\hline 0-1 (referent) & 1.00 & 1.00 & 1.00 \\
\hline $2-4$ & $0.62(0.58-0.65)$ & $0.72(0.68-0.76)$ & $0.87(0.81-0.93)$ \\
\hline $5-10$ & $0.56(0.53-0.59)$ & $0.66(0.62-0.72)$ & $0.83(0.77-0.89)$ \\
\hline$\geq 11$ & $0.55(0.51-0.58)$ & $0.60(0.56-0.64)$ & $0.83(0.76-0.89)$ \\
\hline \multicolumn{4}{|l|}{ Age at diagnosis } \\
\hline $67-75$ y (referent) & & 1.00 & 1.00 \\
\hline $76-85$ y & & $1.36(1.30-1.43)$ & $1.28(1.21-1.35)$ \\
\hline$\geq 86 y$ & & $2.19(2.05-2.33)$ & $1.85(1.71-2.00)$ \\
\hline \multicolumn{4}{|l|}{ Race/ethnicity } \\
\hline $\begin{array}{l}\text { White, non-Hispanic } \\
\text { (referent) }\end{array}$ & & 1.00 & 1.00 \\
\hline Black, non-Hispanic & & $1.15(1.06-1.25)$ & $1.03(0.94-1.14)$ \\
\hline Hispanic & & $1.03(0.93-1.16)$ & $0.91(0.80-1.03)$ \\
\hline $\begin{array}{l}\text { Asian/American Indian/ } \\
\text { Pacific Islander }\end{array}$ & & $0.86(0.74-1.01)$ & $0.97(0.81-1.15)$ \\
\hline Other & & $0.52(0.36-0.77)$ & $1.09(0.94-1.26)$ \\
\hline \multicolumn{4}{|l|}{ Marital status } \\
\hline Married (referent) & & 1.00 & 1.00 \\
\hline Never married & & $1.36(1.25-1.48)$ & $1.22(1.11-1.35)$ \\
\hline Separated/divorced & & $1.35(1.24-1.48)$ & $1.27(1.14-1.40)$ \\
\hline Widowed & & $1.33(1.26-1.40)$ & $1.21(1.14-1.28)$ \\
\hline Unknown & & $1.15(1.03-1.29)$ & $1.09(0.94-1.26)$ \\
\hline \multicolumn{4}{|l|}{$\begin{array}{l}\text { Education level of } \\
\text { residence }\end{array}$} \\
\hline $\begin{array}{l}\text { Quintile } 1 \text { (lowest) } \\
\text { (referent) }\end{array}$ & & 1.00 & 1.00 \\
\hline Quintile 2 & & $1.02(0.95-1.09)$ & $1.08(0.99-1.16)$ \\
\hline Quintile 3 & & $0.94(0.87-1.01)$ & $0.97(0.89-1.06)$ \\
\hline Quintile 4 & & $0.92(0.84-1.00)$ & $0.98(0.89-1.08)$ \\
\hline Quintile 5 & & $0.88(0.80-0.97)$ & $0.92(0.82-1.03)$ \\
\hline \multicolumn{4}{|l|}{ Income level of residence } \\
\hline $\begin{array}{l}\text { Quintile } 1 \text { (lowest) } \\
\text { (referent) }\end{array}$ & & 1.00 & 1.00 \\
\hline Quintile 2 & & $1.00(0.93-1.07)$ & $0.97(0.90-1.05)$ \\
\hline Quintile 3 & & $1.02(0.95-1.11)$ & $0.96(0.88-1.05)$ \\
\hline Quintile 4 & & $1.00(0.92-1.09)$ & $0.98(0.89-1.08)$ \\
\hline Quintile 5 & & $1.03(0.93-1.14)$ & $1.03(0.92-1.16)$ \\
\hline \multicolumn{4}{|l|}{ MSA of residence } \\
\hline $\begin{array}{l}\text { Large metropolitan } \\
\text { (referent) }\end{array}$ & & 1.00 & 1.00 \\
\hline Metropolitan & & $0.99(0.92-1.06)$ & $0.98(0.90-1.06)$ \\
\hline Urban & & $0.95(0.85-1.06)$ & $0.97(0.86-1.10)$ \\
\hline Less urban & & $0.92(0.82-1.02)$ & $0.96(0.84-1.09)$ \\
\hline \multirow[t]{2}{*}{ Rural } & & $0.78(0.65-0.94)$ & $0.81(0.66-1.00)$ \\
\hline & & & continued \\
\hline
\end{tabular}

and additional benefits, which is consistent with results from our prior study in patients with colorectal cancer. ${ }^{79}$

The nation's primary care workforce has been in decline during the past few decades, potentially threatening health conditions, such as cancer, that are sensitive to primary care services. ${ }^{24,81}$ Although primary care physician supply may be an important predictor of health outcomes, effective primary care may well require more than just a sufficient number of primary care physicians. The best evidence for improved quality appears to come from a more fundamental primary care orientation of the health care system. ${ }^{82}$

This study has a number of limitations that should be considered when interpreting our results. First, this study was limited to patients aged 67 years and older having Medicare fee-for-service insurance, and findings may be different for other populations. Selection bias might have affected our results if persons with unknown disease staging were more likely to have late-stage disease. Given that unknown stage was more common for persons with low primary care utilization, excluding patients with an unknown stage from the analysis would bias our findings toward the null (our findings would be conservative). Selection bias could also affect our results of survival (overestimating associations) if healthy persons are more likely to see primary care physicians. We included influenza vaccination in our models as a proxy for this healthy user effect.

The association between visits to a primary care physician and breast cancer mortal- 
ity could also be, in part, the result of lead time bias in which persons receive an earlier disease diagnosis but

Table 4. Adjusted Hazard Ratios for Breast Cancer Mortality $(n=90,537)$ (continued)

\begin{tabular}{|c|c|c|c|}
\hline Characteristic & $\begin{array}{c}\text { Unadjusted } \\
\text { Hazard Ratio } \\
(95 \% \mathrm{Cl})\end{array}$ & $\begin{array}{c}\text { Adjusted Excluding } \\
\text { Stage and Tumor } \\
\text { Size }^{\mathrm{a}} \text { Hazard Ratio } \\
(95 \% \mathrm{Cl})\end{array}$ & $\begin{array}{c}\text { Adjusted Including } \\
\text { Stage and Tumor } \\
\text { Size }^{\text {a }} \text { Hazard Ratio } \\
(95 \% \mathrm{Cl})\end{array}$ \\
\hline \multicolumn{4}{|l|}{ Year of diagnosis } \\
\hline 1994-1997 (referent) & & 1.00 & 1.00 \\
\hline $1998-2000$ & & $1.05(0.99-1.11)$ & $0.97(0.91-1.04)$ \\
\hline $2001-2005$ & & $1.06(1.00-1.13)$ & $0.93(0.87-1.00)$ \\
\hline \multicolumn{4}{|l|}{ Histology type } \\
\hline Ductal (referent) & & 1.00 & 1.00 \\
\hline Lobular & & $0.79(0.74-0.84)$ & $0.88(0.82-0.94)$ \\
\hline Ductal/lobular & & $1.09(0.78-1.51)$ & $1.18(0.83-1.68)$ \\
\hline Favorable & & $0.29(0.24-0.34)$ & $0.45(0.38-0.54)$ \\
\hline Unfavorable & & $1.98(1.78-2.21)$ & $1.10(0.89-1.35)$ \\
\hline Undefined & & $0.88(0.78-1.00)$ & $1.09(0.95-1.25)$ \\
\hline \multicolumn{4}{|l|}{ Tumor grade } \\
\hline $\begin{array}{l}\text { Well differentiated } \\
\text { (referent) }\end{array}$ & & 1.00 & 1.00 \\
\hline $\begin{array}{l}\text { Moderately } \\
\text { differentiated }\end{array}$ & & $2.16(1.96-2.39)$ & $1.66(1.49-1.85)$ \\
\hline Poorly differentiated & & $4.62(4.19-5.10)$ & $2.78(2.50-3.10)$ \\
\hline Undifferentiated & & $4.73(4.04-5.53)$ & $2.71(2.28-3.23)$ \\
\hline Unknown & & $5.07(4.58-5.62)$ & $2.23(1.98-2.51)$ \\
\hline \multicolumn{4}{|l|}{ Estrogen receptor status } \\
\hline $\begin{array}{l}\text { Positive/borderline } \\
\text { (referent) }\end{array}$ & & 1.00 & 1.00 \\
\hline Negative & & $1.52(1.41-1.64)$ & $1.62(1.49-1.75)$ \\
\hline Unknown & & $1.36(1.13-1.64)$ & $1.37(1.09-1.71)$ \\
\hline \multicolumn{4}{|l|}{$\begin{array}{l}\text { Progesterone receptor } \\
\text { status }\end{array}$} \\
\hline $\begin{array}{l}\text { Positive/borderline } \\
\text { (referent) }\end{array}$ & & 1.00 & 1.00 \\
\hline Negative & & $1.40(1.31-1.50)$ & $1.37(1.27-1.47)$ \\
\hline Unknown & & $1.21(1.00-1.46)$ & $1.01(0.81-1.27)$ \\
\hline $\begin{array}{l}\text { Tumor size } \\
\text { (continuous, mm) }\end{array}$ & & & $\begin{array}{l}1.006 \\
(1.006-1.006)\end{array}$ \\
\hline \multicolumn{4}{|l|}{ Stage at diagnosis } \\
\hline I (referent) & & - & 1.00 \\
\hline II & & - & $3.38(3.13-3.64)$ \\
\hline III & & - & $8.90(8.12-9.75)$ \\
\hline IV & & - & $32.34(29.51-35.43)$ \\
\hline Unknown & & - & $3.60(3.25-4.00)$ \\
\hline \multicolumn{4}{|l|}{$\begin{array}{l}\text { Charlson comorbidity } \\
\text { index }\end{array}$} \\
\hline 0 (referent) & & 1.00 & 1.00 \\
\hline 1 & & $1.15(1.08-1.21)$ & $1.20(1.13-1.28)$ \\
\hline$\geq 2$ & & $1.51(1.42-1.60)$ & $1.53(1.43-1.64)$ \\
\hline \multicolumn{4}{|l|}{ Influenza vaccination } \\
\hline No (referent) & & 1.00 & 1.00 \\
\hline Yes & & $0.79(0.76-0.83)$ & $0.92(0.87-0.97)$ \\
\hline \multicolumn{4}{|c|}{ MSA = metropolitan statistical area; SEER = Surveillance Epidemiology and End Results. } \\
\hline \multicolumn{4}{|c|}{ Note: Patients with stage 0 carcinoma (in situ) were excluded in the multivariable analysis. } \\
\hline \multicolumn{4}{|c|}{$\begin{array}{l}\text { a Multivariable Cox proportional models also included indicator variables for SEER Registry location (data not } \\
\text { presented). }\end{array}$} \\
\hline
\end{tabular}

do not have improved survival. Our study attempted to address this issue by exploring the hypothesized causal pathway linking greater use of mammography (proven to lower breast cancer mortality) to earlier stage diagnosis and in turn lower breast cancer mortality. Future studies should explore the association between primary care services and population-based measures of breast cancer mortality.

Our study was limited to administrative data contained within the SEER-Medicare database, which may omit important confounders. For example, we did not have information on severity of comorbid illness, which may be associated with mortality. Our measure of primary care utilization was limited, and we did not have detailed information on the content of the primary care relationship. As such, it is uncertain what specific aspects of the primary care relationship are most important to improve breast cancer outcomes. For example, it is uncertain whether the quantity of visits is more important than the type of visits (health maintenance visits for example).

In conclusion, for Medicare beneficiaries with breast cancer, increasing the number of visits to a primary care physician was associated with an increased likelihood of recent mammography, lower odds of late-stage diagnosis, and lower breast cancer and overall mortality. These findings suggest adequate primary medical care may be an important factor in achieving optimal outcomes for patients with a diagnosis of breast cancer.

To read or post commentaries in response to this article, see it online at http://www.annfammed.orgl content/10/5/401. 
Submitted March 23, 2011; submitted, revised, December 8, 2011; accepted January 4, 2012.

Key words: primary care; breast cancer; Medicare

Funding support: This study was supported by the American Cancer Society (RSGHP-08-141-01-CPHPS). This study used the linked Surveillance, Epidemiology, and End Results (SEER) Medicare database. The collection of the California cancer incidence data used in this study was supported by the California Department of Public Health as part of the statewide cancer reporting program mandated by California Health and Safety Code Section 103885; the National Cancer Institute (NCI) Surveillance, Epidemiology and End Results Program under contract N01-PC-35136 awarded to the Northern California Cancer Center, contract N01-PC-35139 awarded to the University of Southern California, and contract N02-PC-15105 awarded to the Public Health Institute; and the Centers for Disease Control and Prevention's National Program of Cancer Registries, under agreement \#U55/CCR921930-02 awarded to the Public Health Institute.

Disclaimer: The interpretation and reporting of these data are the sole responsibility of the authors. The ideas and opinions expressed herein are those of the authors, and endorsement by the State of California, Department of Public Health the National Cancer Institute, and the Centers for Disease Control and Prevention or their Contractors and Subcontractors is not intended nor should be inferred.

Acknowledgment: The authors acknowledge the efforts of the Applied Research Program, NCl; the Office of Research, Development and Information, Center for Medicare \& Medicaid Services; Information Management Services (IMS), Inc; and the SEER Program tumor registries in the creation of the SEER-Medicare database.

The funding body had no role in the study design or conduct; in the data collection, management, analysis, or interpretation; or in the preparation, review, or approval of the manuscript.

\section{References}

1. Fox SA, Siu AL, Stein JA. The importance of physician communication on breast cancer screening of older women. Arch Intern Med. 1994;154(18):2058-2068.

2. Breen N, Kessler L. Changes in the use of screening mammography: evidence from the 1987 and 1990 National Health Interview Surveys. Am J Public Health. 1994;84(1):62-67.

3. NCI BCSC. Screening mammography: A missed clinical opportunity? Results of the $\mathrm{NCl}$ Breast Cancer Screening Consortium and National Health Interview Survey Studies. JAMA. 1990;264(1):54-58.

4. Fox SA, Murata PJ, Stein JA. The impact of physician compliance on screening mammography for older women. Arch Intern Med. 1991; 151(1):50-56.

5. Lewis SF, Jensen NM. Screening sigmoidoscopy. Factors associated with utilization. J Gen Intern Med. 1996;11(9):542-544.

6. Vernon SW. Participation in colorectal cancer screening: a review. J Natl Cancer Inst. 1997;89(19):1406-1422.

7. Taylor V, Lessler D, Mertens K, et al. Colorectal cancer screening among African Americans: the importance of physician recommendation. J Natl Med Assoc. 2003;95(9):806-812.

8. Bazargan M, Bazargan SH, Farooq M, Baker RS. Correlates of cervical cancer screening among underserved Hispanic and AfricanAmerican women. Prev Med. 2004;39(3):465-473.
9. Cole SR, Young GP, Byrne D, Guy JR, Morcom J. Participation in screening for colorectal cancer based on a faecal occult blood test is improved by endorsement by the primary care practitioner. J Med Screen. 2002;9(4):147-152.

10. Stockwell DH, Woo P, Jacobson BC, et al. Determinants of colorectal cancer screening in women undergoing mammography. Am J Gastroenterol. 2003;98(8):1875-1880.

11. Phillips KA, Haas JS, Liang SY, et al. Are gatekeeper requirements associated with cancer screening utilization? Health Serv Res. 2004; 39(1):153-178.

12. Haggstrom DA, Phillips KA, Liang SY, Haas JS, Tye S, Kerlikowske K. Variation in screening mammography and Papanicolaou smear by primary care physician specialty and gatekeeper plan (United States). Cancer Causes Control. 2004;15(9):883-892.

13. Mclsaac WJ, Fuller-Thomson E, Talbot Y. Does having regular care by a family physician improve preventive care? Can Fam Physician. 2001:47:70-76.

14. Ferrante JM, Rovi S, Das K, Kim S. Family physicians expedite diagnosis of breast disease in urban minority women. J Am Board Fam Med. 2007;20(1):52-59.

15. Barnett PG, Midtling JE. Public policy and the supply of primary care physicians. JAMA. 1989;262(20):2864-2868.

16. Whitcomb ME, Cohen JJ. The future of primary care medicine. N Engl J Med. 2004;351(7):710-712.

17. Rivo ML, Satcher D. Improving access to health care through physician workforce reform. Directions for the 21st century. JAMA. 1993;270(9):1074-1078.

18. Kindig DA, Cultice JM, Mullan F. The elusive generalist physician. Can we reach a 50\% goal? JAMA. 1993;270(9):1069-1073.

19. Colwill JM. Where have all the primary care applicants gone? N Engl J Med. 1992;326(6):387-393.

20. Newton DA, Grayson MS. Trends in career choice by US medical school graduates. JAMA. 2003;290(9):1179-1182.

21. Pugno PA, McPherson DS, Schmittling GT, Kahn NB Jr. Results of the 2003 National Resident Matching Program: family practice. Fam Med. 2003;35(8):564-572.

22. Jeffe DB, Whelan AJ, Andriole DA. Primary care specialty choices of United States medical graduates, 1997-2006. Acad Med. 2010;85(6) 947-958.

23. Dentzer S. Reinventing primary care: a task that is far 'too important to fail'. Health Aff (Millwood). 2010;29(5):757.

24. Bodenheimer T, Pham HH. Primary care: current problems and proposed solutions. Health Aff (Millwood). 2010;29(5):799-805.

25. Starfield B, Shi L, Grover A, Macinko J. The effects of specialist supply on populations' health: assessing the evidence. Health Aff (Millwood). 2005;Jan-Jun;Suppl Web Exclusives:W5-97-W5-107.

26. Shi L, Macinko J, Starfield B, et al. Primary care, infant mortality, and low birth weight in the states of the USA. J Epidemiol Community Health. 2004;58(5):374-380.

27. Roetzheim RG, Pal N, Tennant C, et al. Effects of health insurance and race on early detection of cancer. J Natl Cancer Inst. 1999;91(16):1409-1415.

28. Roetzheim RG, Gonzalez EC, Ferrante JM, Pal N, Van Durme DJ, Krischer JP. Effects of health insurance and race on breast carcinoma treatments and outcomes. Cancer. 2000;89(11):2202-2213.

29. Ferrante JM, Gonzalez EC, Pal N, Roetzheim RG. Effects of physician supply on early detection of breast cancer. J Am Board Fam Pract. 2000;13(6):408-414.

30. Roetzheim RG, Gonzalez EC, Ramirez A, Campbell R, van Durme DJ. Primary care physician supply and colorectal cancer. J Fam Pract. 2001;50(12):1027-1031. 
31. Campbell RJ, Ramirez AM, Perez K, Roetzheim RG. Cervical cancer rates and the supply of primary care physicians in Florida. Fam Med 2003;35(1):60-64

32. Shi L, Macinko J, Starfield B, Xu J, Politzer R. Primary care, income inequality, and stroke mortality in the United States: a longitudinal analysis, 1985-1995. Stroke. 2003;34(8):1958-1964.

33. Shi L, Starfield B, Kennedy B, Kawachi I. Income inequality, primary care, and health indicators. J Fam Pract. 1999;48(4):275-284.

34. Parsons MA, Askland KD. Cancer of the colorectum in Maine, 1995 1998: determinants of stage at diagnosis in a rural state. J Rural Health. 2007;23(1):25-32.

35. Macinko J, Starfield B, Shi L. Quantifying the health benefits of primary care physician supply in the United States. Int J Health Serv. 2007;37(1):111-126.

36. Shi L, Macinko J, Starfield B, Politzer R, Wulu J, Xu J. Primary care, social inequalities and all-cause, heart disease and cancer mortality in US counties: a comparison between urban and non-urban areas. Public Health. 2005;119(8):699-710.

37. Davidson PL, Bastani R, Nakazono TT, Carreon DC. Role of community risk factors and resources on breast carcinoma stage at diagno sis. Cancer. 2005;103(5):922-930.

38. Van Durme DJ, Ullman R, Campbell RJ, Roetzheim R. Effects of physician supply on melanoma incidence and mortality in Florida. South Med J. 2003;96(7):656-660.

39. Trimble CL, Richards LA, Wilgus-Wegweiser B, Plowden K, Rosenthal DL, Klassen A. Effectiveness of screening for cervical cancer in an inpatient hospital setting. Obstet Gynecol. 2004;103(2):310-316.

40. Klassen A, Hall A, Bowie J, Weisman CS. Improving cervical cancer screening in hospital settings. Prev Med. 2000;31(5):538-546.

41. Freeman HJ. Documentation of rectal examination performance in the clinical teaching unit of a university hospital. Can J Gastroenterol. 2000;14(4):272-276.

42. Sharma VK, Komanduri S, Nayyar $S$, et al. An audit of the utility of in-patient fecal occult blood testing. Am J Gastroenterol. 2001;96(4): 1256-1260.

43. Brull R, Ghali WA, Quan H. Missed opportunities for prevention in general internal medicine. [see comment]. CMAJ. 1999;160(8):11371140.

44. White MJ. Consideration of women's health issues by housestaff performing admitting histories and physical examinations. Acad Med. 1993;68(9):698-700.

45. Ziffer A, Song P, Mandelblatt J. Preventive health practices in a teaching hospital: house staff attitudes and performance of gynecological screening. Am J Prev Med. 1987;3(3):142-146.

46. Van Harrison R, Janz NK, Wolfe RA, et al. Characteristics of primary care physicians and their practices associated with mammography rates for older women. Cancer. 2003;98(9):1811-1821.

47. Harrison RV, Janz NK, Wolfe RA, Tedeschi PJ, Huang X, McMahon LF Jr. 5-Year mammography rates and associated factors for older women. Cancer. 2003;97(5):1147-1155.

48. Huth JF, O'Connor AP. Breast cancer screening practices for hospitalized women. J Cancer Educ. 1995;10(1):22-24.

49. Yu X, McBean AM, Virnig BA. Physician visits, patient comorbidities, and mammography use among elderly colorectal cancer survivors. J Cancer Surviv. 2007;1(4):275-282.

50. Baldwin LM, Adamache W, Klabunde CN, Kenward K, Dahlman C L. Warren J. Linking physician characteristics and medicare claims data: issues in data availability, quality, and measurement. Med Care. 2002;40(8 Suppl):IV-82-95.

51. Gornick ME, Eggers PW, Riley GF. Associations of race, education, and patterns of preventive service use with stage of cancer at time of diagnosis. Health Serv Res. 2004;39(5):1403-1427.
52. Ferrante JM, Chen PH, Kim S. The effect of patient navigation on time to diagnosis, anxiety, and satisfaction in urban minority women with abnormal mammograms: a randomized controlled trial. J Urban Health. 2008;85(1):114-124.

53. Charlson ME, Pompei P, Ales KL, MacKenzie CR. A new method of classifying prognostic comorbidity in longitudinal studies: development and validation. J Chronic Dis. 1987;40(5):373-383.

54. Badgwell BD, Giordano SH, Duan ZZ, et al. Mammography before diagnosis among women age 80 years and older with breast cancer. J Clin Oncol. 2008;26(15):2482-2488.

55. Freeman JL, Klabunde CN, Schussler N, Warren JL, Virnig BA, Cooper GS. Measuring breast, colorectal, and prostate cancer screening with medicare claims data. Med Care. 2002;40(8) (Suppl):IV-36-IV-42.

56. Randolph WM, Mahnken JD, Goodwin JS, Freeman JL. Using Medicare data to estimate the prevalence of breast cancer screening in older women: comparison of different methods to identify screening mammograms. Health Serv Res. 2002;37(6):1643-1657.

57. Ferrante JM, Gonzalez EC, Pal N, Roetzheim RG. Effects of physician supply on early detection of breast cancer. J Am Board Fam Pract. 2000;13(6):408-414.

58. Gorey KM, Luginaah IN, Holowaty EJ, Fung KY, Hamm C. Associations of physician supplies with breast cancer stage at diagnosis and survival in Ontario, 1988 to 2006. Cancer. 2009;115(15):3563-3570.

59. Gorey KM, Luginaah IN, Fung KY, et al. Physician supply and breast cancer survival. J Am Board Fam Med. 2010;23(1):104-108.

60. Fleisher JM, Lou JQ, Farrell M. Relationship between physician supply and breast cancer survival: a geographic approach. J Community Health. 2008;33(4):179-182.

61. Keating NL, Landrum MB, Ayanian JZ, Winer EP, Guadagnoli E. The association of ambulatory care with breast cancer stage at diagnosis among Medicare beneficiaries. J Gen Intern Med. 2005;20(1):38-44.

62. Hawley ST, Earp JA, O'Malley M, Ricketts TC. The role of physician recommendation in women's mammography use: is it a 2-stage process? Med Care. 2000;38(4):392-403.

63. Meissner HI, Breen N, Taubman ML, Vernon SW, Graubard BI. Which women aren't getting mammograms and why? (United States). Cancer Causes Control. 2007;18(1):61-70.

64. Shi L, Starfield B. Primary care, income inequality, and self-rated health in the United States: a mixed-level analysis. Int J Health Serv. 2000;30(3):541-555.

65. Hahn KME, Bondy ML, Selvan M, et al. Factors associated with advanced disease stage at diagnosis in a population-based study of patients with newly diagnosed breast cancer. Am J Epidemiol. 2007;166(9):1035-1044

66. McEligot AJ, Im T, Dillman RO, et al. Abstracting height and weight from medical records, and breast cancer pathologic factors. Cancer Causes Control. 2008;19(10):1217-1226

67. Jang TL, Bekelman JE, Liu Y, et al. Physician visits prior to treatment for clinically localized prostate cancer. Arch Intern Med. 2010;170(5):440-450

68. Smith GF, Toonen TR. The role of the primary care physician during the active treatment phase. Prim Care. 2009;36(4):685-702.

69. Bober SL, Recklitis CJ, Campbell EG, et al. Caring for cancer survivors: a survey of primary care physicians. Cancer. 2009;115(18) (Suppl):4409-4418

70. Klabunde CN, Ambs A, Keating NL, et al. The role of primary care physicians in cancer care. J Gen Intern Med. 2009;24(9):1029-1036.

71. Hickner J, Kent S, Naragon P, Hunt L. Physicians' and patients' views of cancer care by family physicians: a report from the American Academy of Family Physicians National Research Network. Fam Med. 2007;39(2):126-131. 
72. Pascoe SW, Neal RD, Allgar VL, Selby PJ, Wright EP. Psychosocial care for cancer patients in primary care? Recognition of opportunities for cancer care. Fam Pract. 2004;21(4):437-442.

73. Hollowell K, Olmsted CL, Richardson AS, et al. American Society of Clinical Oncology-recommended surveillance and physician specialty among long-term breast cancer survivors. Cancer. 2010.

74. Field TS, Doubeni C, Fox MP, et al. Under utilization of surveillance mammography among older breast cancer survivors. J Gen Intern Med. 2008;23(2):158-163.

75. Earle CC, Burstein HJ, Winer EP, Weeks JC. Quality of non-breast cancer health maintenance among elderly breast cancer survivors. J Clin Oncol. 2003;21(8):1447-1451

76. Ferrante JM, Balasubramanian BA, Hudson SV, Crabtree BF. Principles of the patient-centered medical home and preventive services delivery. Ann Fam Med. 2010;8(2):108-116.

77. Ogle KS, Swanson GM, Woods N, Azzouz F. Cancer and comorbidity: redefining chronic diseases. Cancer. 2000;88(3):653-663.
78. Roetzheim RG, Pal N, Gonzalez EC, et al. The effects of physician supply on the early detection of colorectal cancer. J Fam Pract. 1999;48(11):850-858

79. Ferrante JM, McCarthy EP, Gonzalez EC, et al. Primary care utilization and colorectal cancer outcomes among Medicare beneficiaries. Arch Intern Med. 2011;171(19):1747-1757.

80. Rosenblatt RA, Hart LG, Baldwin LM, Chan L, Schneeweiss R. The generalist role of specialty physicians: is there a hidden system of primary care? JAMA. 1998;279(17):1364-1370.

81. US Department of Health and Human Services. Healthy People 2010. 2nd ed. With Understanding and Improving Health and Objectives for Improving Health. Washington, DC: US Government Printing Office, 2000.

82. Friedberg MW, Hussey PS, Schneider EC. Primary care: a critical review of the evidence on quality and costs of health care. Health Aff (Millwood). 2010;29(5):766-772.

Please complete this form and mail to the following address or fax to Annals Circulation at 913-906-6080:

Annals of Family Medicine, Circulation Department, 11400 Tomahawk Creek Pkwy, Leawood, KS 66211-2680

Check if member of sponsoring organization:

\section{AAFP}

$\square$ AFMRD $\square \mathrm{ABFM}$

$\square$ NAPCRG

STFM

$\square$ ADFM

ID number from label on your journal cover

OLD Information (Please print.)

$$
\text { Name }
$$

Company (if applicable)

Address (Street plus Apt or Ste)

City

Country

Postal Code (9-digit ZIP for US)

Telephone

E-Mai
NEW Information (Please print.)

\begin{tabular}{ll}
\hline Name \\
\hline Company (if applicable) & \\
\hline Address (Street plus Apt or Ste) & \\
\hline City & Postal Code (9-digit ZIP for US) \\
\hline Country & \\
\hline Telephone & Fax \\
\hline E-Mail
\end{tabular}

